\title{
ORIGINAL RESEARCH \\ Practical Scoring System for the Identification of Patients with Intracerebral Hemorrhage at Highest Risk of Harboring an Underlying Vascular Etiology: The Secondary Intracerebral Hemorrhage Score
}

J.E. Delgado Almandoz

P.W. Schaefer

J.N. Goldstein

J. Rosand

M.H. Lev

R.G. González

J.M. Romero

\begin{abstract}
BACKGROUND AND PURPOSE: An ICH patient's risk of harboring an underlying vascular etiology varies according to baseline clinical and NCCT characteristics. Our aim was to develop a practical scoring system to stratify patients with $\mathrm{ICH}$ according to their risk of harboring a vascular etiology.

MATERIALS AND METHODS: Using a data base of 623 patients with ICH evaluated with MDCTA during a 9-year period, we developed a scoring system based on baseline clinical characteristics (age group [0-2 points], sex [0-1 point], neither known HTN nor impaired coagulation [0-1 point]), and NCCT categorization (0-2 points) to predict the risk of harboring a vascular lesion as the $\mathrm{ICH}$ etiology ( $\mathrm{SICH}$ score). We subsequently applied the SICH score to a prospective cohort of 222 patients with ICH who presented to our emergency department during a 13-month period. Using ROC analysis, we calculated the AUC and MOP for the SICH score in both the retrospective and prospective patient cohorts separately and the entire patient population. Patients with $\mathrm{SAH}$ in the basal cisterns were excluded.
\end{abstract}

RESULTS: A vascular etiology was found in 120 of 845 patients with ICH evaluated with MDCTA (14.2\%), most commonly AVMs (45.8\%), aneurysms with purely intraparenchymal rupture (21.7\%), and DVSTs (16.7\%). The MOP was reached at a $\mathrm{SICH}$ score of $>2$, with the highest incidence of vascular ICH etiologies in patients with $\mathrm{SICH}$ scores of 3 (18.5\%), 4 (39\%), 5 (84.2\%), and 6 (100\%). There was no significant difference in the AUC between both patient cohorts $(0.86-0.87)$.

CONCLUSIONS: The SICH score successfully predicts a given $\mathrm{ICH}$ patient's risk of harboring an underlying vascular etiology and could be used as a guide to select patients with $\mathrm{ICH}$ for neurovascular evaluation to exclude the presence of a vascular abnormality.

\begin{abstract}
ABBREVIATIONS: aPTT = activated partial thromboplastin time; $\mathrm{AUC}=$ area under the curve; $\mathrm{AVF}=$ arteriovenous fistula; $\mathrm{AVM}=$ arteriovenous malformation; $\mathrm{Cl}=$ confidence interval; $\mathrm{CTA}=$ $\mathrm{CT}$ angiogram; DVST = dural venous sinus thrombosis; $\mathrm{HTN}=$ hypertension; $\mathrm{ICH}=$ intracerebral hemorrhage; INR = international normalized ratio; IVH = intraventricular hemorrhage; MDCTA = multidetector $\mathrm{CT}$ angiography; MIP = maximum intensity projection; $\mathrm{MOP}=$ maximum operating point; $\mathrm{NCCT}=$ noncontrast $\mathrm{CT}$; ROC = receiver operating characteristic analysis; $\mathrm{SAH}=$ subarachnoid hemorrhage; $\mathrm{SICH}=$ secondary $\mathrm{ICH}$
\end{abstract}

$\mathbf{N}$ ontraumatic ICH accounts for 10\%-15\% of cases of acute stroke. ${ }^{1}$ Although in most cases the ICH is caused by hypertension, amyloid angiopathy, or impaired coagulation, historical features such as hypertension, older age, or coagulopa-

Received February 11, 2010; accepted after revision April 4.

From the Division of Neuroradiology (J.E.D.A., P.W.S., M.H.L., R.G.G., J.M.R.), Department of Radiology; Department of Emergency Medicine (J.N.G.); and Department of Neurology and Center for Human Genetic Research (J.R.), Massachusetts General Hospital, Harvard Medical School, Boston, Massachusetts; and Division of Neuroradiology (J.E.D.A.), Mallinckrodt Institute of Radiology, Washington University, St Louis, Missouri. The following sources of funding were used, in part, for the preparation of this manuscript: American Heart Association Grant-in-Aid 0755984T and the National Institutes of Health/ National Institute of Neurological Disorders and Stroke grant K23NS059774.

Paper previously presented in part at: International Stroke Conference, February 24, 2010; San Antonio, Texas; and as a whole at: Annual Meeting of the American Society of Neuroradiology, May 20, 2010; Boston, Massachusetts.

Please address correspondence to Josser E. Delgado Almandoz, MD, Division of Neuroradiology, Mallinckrodt Institute of Radiology, Washington University, Campus Box 8131, 510 S Kingshighway Blvd, St Louis, M0 63110; e-mail: delgadoj@mir.wustl.edu

Indicates open access to non-subscribers at www.ajnr.org

DOI 10.3174/ajnr.A2156 thy, are often not enough, in isolation, to establish the ICH etiology. Indeed, in many cases, the ICH is due to vascular lesions such as AVMs, intracranial aneurysms with purely intraparenchymal rupture, and DVST (ie, SICH)..$^{1-3}$ Timely and accurate identification of patients with SICH is important because therapeutic options and rates of rehemorrhage are substantially different from primary ICH. ${ }^{1,2,4-6}$

Due to its widespread availability, rapidity of acquisition, lower cost, and favorable risk profile compared with conventional catheter angiography, MDCTA is rapidly becoming the favored diagnostic examination in the initial evaluation of patients presenting to the emergency department with $\mathrm{ICH}$ at many medical centers in the United States and around the world. In addition, several prior studies have demonstrated that compared with conventional catheter angiography and intraoperative findings, MDCTA is highly accurate for the detection of underlying vascular lesions in patients with $\mathrm{ICH}$, with reported sensitivities ranging from $89 \%$ to $96 \%$ and specificities of $92 \%$ to $100 \% .^{7-10}$

A prior study of 623 patients with $\mathrm{ICH}$ examined with MDCTA at our institution identified clinical and NCCT char- 
acteristics that independently predicted an increased incidence of a vascular lesion as the ICH etiology: age younger than 46 years (47\%), neither known hypertension nor impaired coagulation at presentation (33\%), lobar (20\%) or infratentorial (16\%) ICH location, and female sex $(18 \%){ }^{10}$ These findings are similar to those of several prior studies of patients with ICH examined with conventional catheter angiography. ${ }^{11-20}$ However, to date, no comprehensive system for the stratification of patients with ICH according to their risk of harboring an underlying vascular etiology has been developed. Indeed, the need for such a risk-stratification system and a standardized approach to the diagnostic work-up of this patient population is underscored by a recent literature review and multinational physician survey conducted at several leading medical institutions in Europe. ${ }^{21}$

The purpose of this study was to develop a practical scoring system to predict a given ICH patient's risk of harboring an underlying vascular lesion as the ICH etiology.

\section{Materials and Methods}

\section{Patient Selection}

Our study was approved by the institutional review board of our hospital and was conducted in compliance with the Health Insurance Portability and Accountability Act. First, we re-analyzed a retrospective cohort of 623 consecutive patients who presented to our emergency department from January 1, 2000, to November 1, 2008, with the following inclusion criteria: 1) age 18 years or older, 2) evidence of nontraumatic ICH on a NCCT examination of the head, and 3) evaluation with a CTA of the intracranial circulation within 24 hours of presentation. Subsequently, we conducted a prospective observational study of a cohort of patients who presented to our emergency department from November 2, 2008, to December 13, 2009, with the same inclusion criteria detailed above.

Patient exclusion criteria for both cohorts were the following: 1) the presence of associated SAH in the basal cisterns, 2) loss of graywhite matter differentiation in a vascular territory indicating a preestablished acute ischemic stroke, 3) a known intracranial vascular abnormality or mass lesion, or 4) known probable cerebral amyloid angiopathy according to the Boston criteria. ${ }^{22}$

\section{Image Acquisition}

NCCT and MDCTA examinations were performed according to standard protocols on 16- or 64-section helical CT scanners. The NCCT examination was performed by using an axial technique with $120-$ $140 \mathrm{kVp}, 170 \mathrm{~mA}, 2$-second scanning time, and 5-mm-section-thickness reconstruction. MDCTA was subsequently performed by scanning from the base of the $\mathrm{C} 1$ vertebral body to the vertex by using the following parameters: pitch, 0.5 ; collimation, $1.25 \mathrm{~mm}$; maximal $\mathrm{mA}$, 350 ; kVp 120 ; FOV, $22 \mathrm{~cm}$; and $65-85 \mathrm{~mL}$ of nonionic contrast material administered by a power injector at $4-5 \mathrm{~mL}$ per second into an antecubital vein with either a fixed 25-second delay between the onset of contrast injection and the start of scanning (the delay was increased to 40 seconds in patients with atrial fibrillation) or SmartPrep, a semiautomatic contrast bolus triggering technique (GE Healthcare, Milwaukee, Wisconsin). All patients in the prospective cohort were scanned by using SmartPrep. The resulting 1.25-mm-thick axial source images were digitally archived. Standard MIP images of the major intracranial vessels were created by the $3 \mathrm{D}$ laboratory. The decision to perform MDCTA was at the discretion of the clinical providers.

\section{Image Analysis}

The image analysis methodology in the retrospective patient cohort has been previously published..$^{10}$ In the prospective patient cohort, the NCCTs were reviewed by 2 neuroradiologists blinded to the clinical data and final diagnosis, to determine the presence of associated SAH and NCCT categorization as detailed below. All differences in reader interpretations for the NCCT categorization were resolved by consensus. At least 2 weeks after review of the NCCT, the CTA source and MIP images were reviewed by the same 2 neuroradiologists, blinded to the NCCT categorization, clinical data, and final diagnosis, to determine, by consensus reading, the presence of an underlying vascular etiology for the $\mathrm{ICH}$.

A high-probability NCCT was defined as an examination in which there were either 1) enlarged vessels or calcifications along the margins of the ICH or 2) hyperattenuation within a dural venous sinus or cortical vein along the presumed venous drainage path of the ICH. A low-probability NCCT was defined as an examination in which none of the findings of a high-probability NCCT were present and the ICH was located within the basal ganglia, thalamus, or brain stem. An indeterminate NCCT was defined as an examination that did not meet criteria for a high- or low-probability NCCT (most commonly, lobar or cerebellar ICH).

A positive CTA was defined as a study in which an underlying vascular lesion as the ICH etiology was identified. For the diagnosis of DVST and/or cortical vein thrombosis, nonopacification of the affected venous structure on the first-pass CTA was confirmed by either persistent nonopacification on delayed images (if obtained) or corresponding hyperattenuation in the NCCT examination.

\section{Medical Record Review}

Medical records were reviewed for patient age, sex, known hypertension, and impaired coagulation. Patients were classified as hypertensive if they had a known history of hypertension or were taking antihypertensive medications at presentation. Patients were classified as having impaired coagulation if, at presentation, they were receiving daily antiplatelet therapy with aspirin or clopidogrel, had a platelet count of $<50,000$ cells per cubic mm of blood, had an INR of $>3.0,{ }^{23}$ or had an aPTT of $>80$ seconds.

\section{Statistical Analysis}

Statistical analysis was performed using the MedCalc package for Windows, Version 11.1 (MedCalc Software, Mariakerke, Belgium). First, we re-analyzed our previously reported retrospective cohort of 623 patients with ICH to include both the ICH location and NCCT categorization in addition to the baseline clinical characteristics in the multivariate logistic regression model to determine the independent predictors of a positive CTA.

Subsequently, we used the independent predictors of a positive CTA to construct a scoring system to predict a given ICH patient's risk of harboring a vascular lesion as the ICH etiology. We then applied this scoring system to both the retrospective derivation and prospective validation patient cohorts and performed ROC analysis to determine the AUC and MOP for the scoring system in both cohorts separately and in the entire patient population. A $P$ value $\leq .05$ was considered statistically significant. 


\begin{tabular}{|c|c|c|c|c|c|c|}
\hline \multirow[b]{2}{*}{ Etiology } & \multicolumn{2}{|c|}{$\begin{array}{c}\text { Retrospective } \\
\text { Cohort } \\
(n=91)\end{array}$} & \multicolumn{2}{|c|}{$\begin{array}{c}\text { Prospective } \\
\text { Cohort } \\
(n=29)\end{array}$} & \multicolumn{2}{|c|}{$\begin{array}{l}\text { All Patients } \\
(n=120)\end{array}$} \\
\hline & No. & $\%$ & No. & $\%$ & No. & $\%$ \\
\hline$\overline{A V M}$ & 40 & 44 & 15 & 51.7 & 55 & 45.8 \\
\hline Aneurysm & $21^{a}$ & 23 & 5 & 17.2 & $26^{a}$ & 21.7 \\
\hline DVST & 17 & 18.7 & 3 & 10.3 & 20 & 16.7 \\
\hline AVF & 8 & 8.8 & 3 & 10.3 & 11 & 9.2 \\
\hline Vasculopathy & 3 & 3.3 & $1^{\mathrm{b}}$ & 3.5 & $4^{b}$ & 3.3 \\
\hline Moyamoya & 2 & 2.2 & 2 & 7 & 4 & 3.3 \\
\hline
\end{tabular}

a Includes 3 pseudoaneurysms.

Includes a patient in whom vasculitis led to pseudoaneurysm formation and rupture.

\section{Results}

The baseline clinical and radiologic characteristics of the 623 patients included in our retrospective cohort have been published previously. ${ }^{10}$ From November 2, 2008, to December 13, 2009, a total of 253 adult patients presented to our emergency department with ICH on an NCCT exami- nation and were evaluated with a CTA of the intracranial circulation within 24 hours of presentation. Thirty-one patients were excluded from the study $(12.3 \%)$ : 14 due to the presence of associated SAH within the basal cisterns ( 9 of which had an underlying vascular etiology [64.3\%]), 8 due to a known vascular or mass lesion, 7 due to loss of graywhite matter differentiation in a vascular territory indicating a pre-established acute ischemic stroke, and 2 due to known amyloid angiopathy.

Hence, a total of 222 patients met the inclusion criteria of the prospective cohort, with a mean age of 67 years (range, $18-94$ years), 111 of which were men (50\%) and 111 , women $(50 \%)$. The number of patients with $\mathrm{ICH}$ evaluated with MDCTA constituted approximately $50.8 \%$ of the total number of patients with nontraumatic ICH presenting to our emergency department during the time period of the retrospective cohort and $77.8 \%$ of the patients with nontraumatic ICH presenting to our emergency department during to the time period of the prospective cohort.
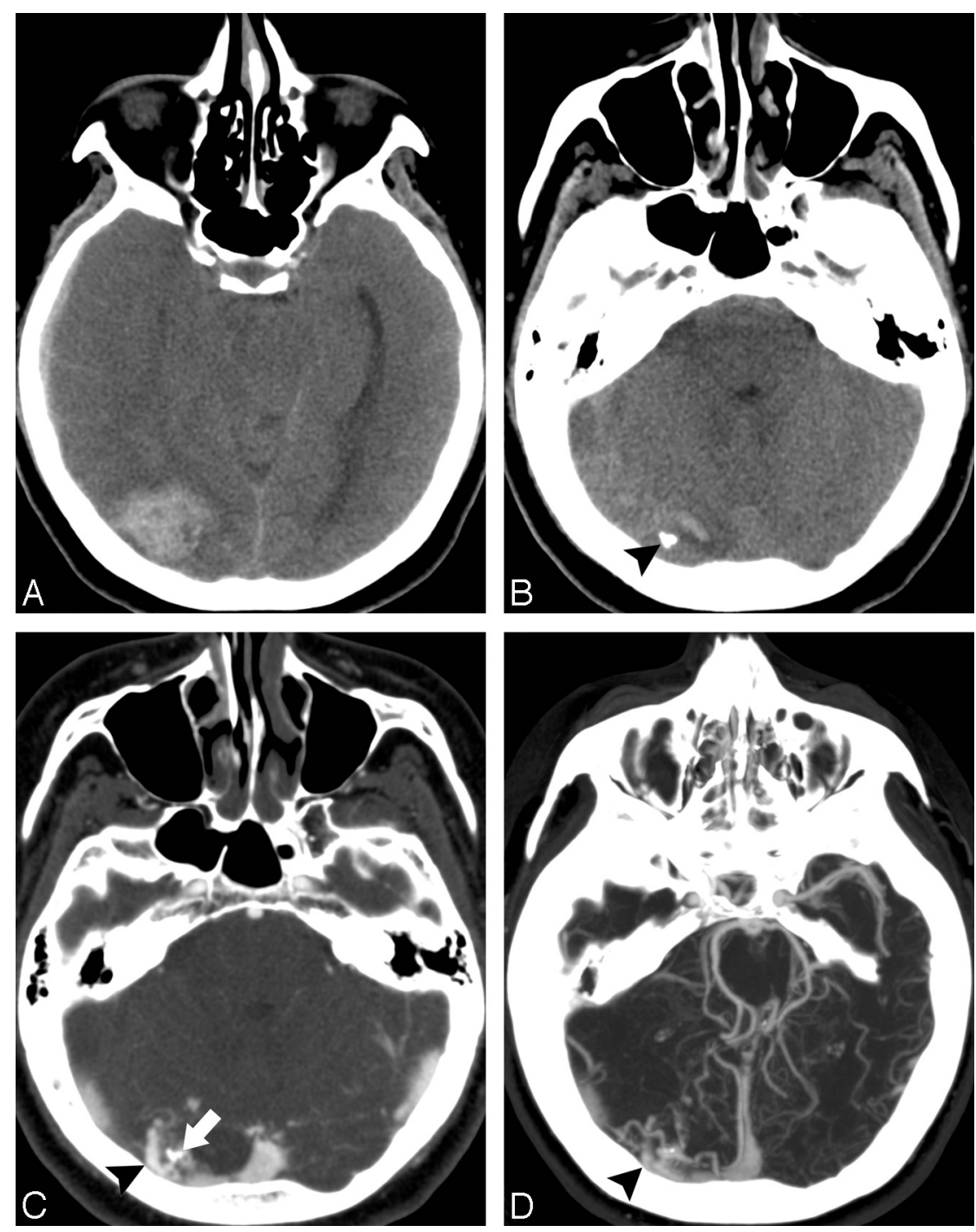

Fig 1. A 45-year-old woman without a history of hypertension and with intact coagulation presented with acute onset of headache and visual changes. $A$ and $B$, High-probability NCCT scan demonstrates an acute right occipital ICH with calcifications along its posteroinferior margin (arrowhead, B; SICH score, 6). There was associated subdural hemorrhage overlying the right temporal lobe but no associated IVH or SAH. C, CTA source image demonstrates a tangle of abnormal vessels along the posteroinferior aspect of the ICH (arrowhead) with associated calcifications (arrow), consistent with an AVM. D, CTA MIP image in the axial plane redemonstrates the right occipital AVM (arrowhead) with arterial supply from branches of the right posterior cerebral artery and drainage to the right transverse sinus. 

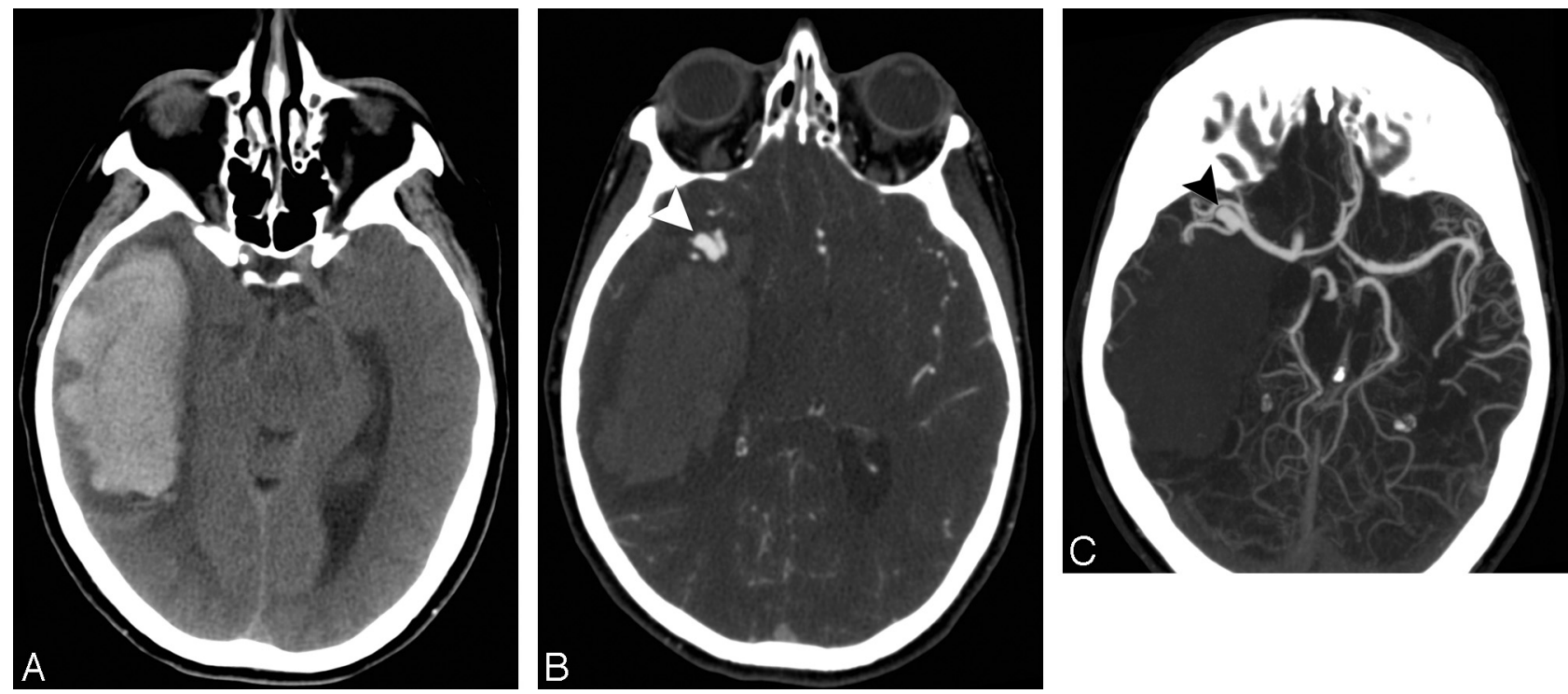

Fig 2. A 50-year-old woman with a history of hypertension and intact coagulation presented with acute onset of left-sided weakness. $A$, Indeterminate NCCT scan demonstrates an acute right temporal ICH without associated IVH or SAH (SICH score, 3). B, CTA source image demonstrates an 11-mm outpouching arising from the right MCA bifurcation (arrowhead), consistent with an aneurysm. C, CTA MIP image in the axial plane redemonstrates the right MCA bifurcation aneurysm (arrowhead).

\section{Vascular ICH Etiologies in the Patient Population}

Table 1 depicts the frequency of the different vascular ICH etiologies in both patient cohorts separately as well as in the entire patient population. MDCTA demonstrated a vascular ICH etiology in 29 of the 222 patients included in the prospective patient cohort (13.1\%). Overall, an underlying vascular etiology for the ICH was identified in 120 of the 845 patients in the entire patient population (14.2\%).

In the prospective patient cohort, the most common vascular etiologies for the ICH were AVMs (15, 51.7\%; Fig 1), aneurysms with purely intraparenchymal rupture $(5,17.2 \%$; Fig 2), DVSTs (3, 10.3\%; Fig 3), and AVFs (3, 10.3\%; Fig 4). In the entire patient cohort, the most common vascular ICH etiologies were AVMs $(55,45.8 \%)$, aneurysms with purely intraparenchymal rupture (26, 21.7\%), DVSTs $(20,16.7 \%)$, and AVFs (11,9.2\%). However, in a small number of patients, the ICHs were secondary to vasculopathy (4, 3.3\%; Fig 5) and Moyamoya phenomenon (4, 3.3\%; Fig 6).

\section{NCCT Categorization}

Interobserver agreement for the NCCT categorization in the prospective cohort was almost perfect ( $\kappa$ statistic, $0.97 ; 95 \%$ CI, 0.93-1.0). In this cohort, 80 NCCTs were categorized as low-probability (36\%), 2 of which had a positive CTA $(2.5 \%, 1$ lenticulostriate aneurysm and 1 lenticulostriate pseudoaneurysm secondary to vasculitis); 132 NCCTs were categorized as indeterminate $(59.5 \%), 19$ of which had a positive CTA (14.4\%); and 10 NCCTs were categorized as high-probability $(4.5 \%, 8$ due to enlarged vessels or calcifications along the margins of the ICH and 2 due to hyperattenuation within a dural venous sinus or cortical vein along the presumed venous drainage path of the ICH), 8 of which had a positive CTA $(80 \%)$.

In the entire patient population, 263 NCCTs were categorized as low-probability (31.1\%), 6 of which had a positive CTA $(2.3 \%, 2$ basal ganglia AVMs, 1 thalamic AVM, 1 pontine AVM, 1 lenticulostriate aneurysm, and 1 lenticulostriate pseu- doaneurysm secondary to vasculitis); 553 NCCTs were categorized as indeterminate (65.5\%), 90 of which had a positive CTA (16.3\%); and 29 NCCTs were categorized as high-probability $(3.4 \%, 16$ due to enlarged vessels or calcifications along the margins of the $\mathrm{ICH}$ and 13 due to hyperattenuation within a dural venous sinus or cortical vein along the presumed venous drainage path of the ICH), 24 of which had a positive CTA $(82.8 \%)$.

\section{Re-Analysis of the Multivariate Logistic Regression Model in the Retrospective Cohort}

When the NCCT categorization was added to the previously published multivariate logistic regression model that included the ICH location and baseline clinical characteristics (age group, sex, known hypertension, impaired coagulation, and neither known hypertension nor impaired coagulation at presentation), the following variables became the independent predictors of a positive CTA: age group $(P$ value $<.0001)$, NCCT categorization $(P$ value $<.0001)$, neither known hypertension nor impaired coagulation at presentation $(P$ value $=.014)$, and sex $(P$ value $=.032)$.

\section{The SICH Score}

We used the independent predictors of a positive CTA—age group, NCCT categorization, neither known hypertension nor impaired coagulation at presentation, and sex-to construct a practical scoring system to predict a given ICH patient's risk of harboring a vascular lesion as the ICH etiology and designated it the SICH score (Table 2).

The results of the application of this scoring system to both the retrospective-derivation and prospective-validation patient cohorts, as well as to the entire patient population, are shown in Table 3. The SICH score successfully predicted a given ICH patient's risk of harboring a vascular etiology in each patient cohort separately as well as in the entire patient population, with the highest incidences of vascular lesions ob- 

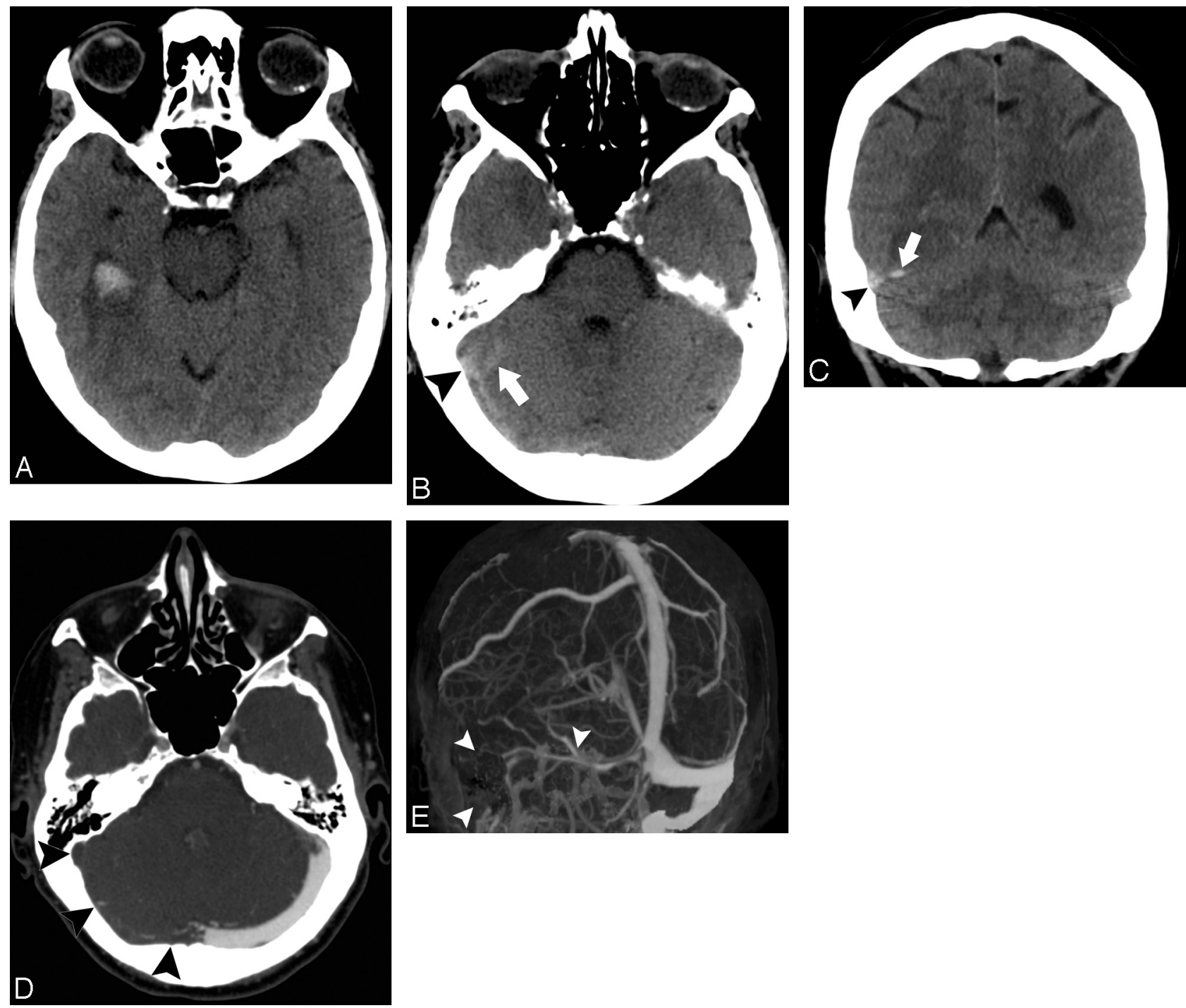

Fig 3. A 60-year-old woman without a history of hypertension and with intact coagulation presented with increasing headache during the past several days. $A$ and $B$, High-probability NCCT scan demonstrates an acute right mesiotemporal ICH with subtle associated hyperattenuation within the distal right vein of Labbe (arrow, B) and right transverse sinus (arrowhead, $B$; SICH score,5). C, Coronal NCCT scan reformation improves depiction of the hyperattenuation within the distal right vein of Labbe (arrow) and right transverse sinus (arrowhead). $D$, CT venogram source image obtained immediately after the CTA demonstrates nonopacification of the right transverse and sigmoid sinuses (arrowheads), consistent with DVST. E, CT venogram MIP image after calvarial segmentation redemonstrates the right transverse and sigmoid sinus thrombosis (arrowheads).

served in patients with SICH scores of 3 (18.5\%), 4 (39\%), 5 $(84.2 \%)$, and $6(100 \%)$.

In ROC analysis, there was no significant difference in the AUCs between the retrospective $(0.86 ; 95 \% \mathrm{CI}, 0.83-0.89)$ and prospective patient cohorts $(0.87 ; 95 \% \mathrm{CI}, 0.82-0.91)$. In addition, the MOP was reached at a SICH score of $>2$ in both patient cohorts as well as in the entire population, with high sensitivity $(85.8 \%)$ and specificity $(72.3 \%)$ for the detection of the vascular $\mathrm{ICH}$ etiologies present in the entire patient population.

Overall, patients with low SICH scores (0-2) comprised $64 \%$ of patients in our entire population and had a low incidence of vascular etiologies (3.1\%), whereas patients with high SICH scores $(\geq 3)$ comprised $36 \%$ of patients and had a high incidence of vascular etiologies (33.9\%).

\section{Discussion}

We have developed and prospectively validated a practical scoring system that predicts a given ICH patient's risk of har- boring an underlying vascular etiology, the SICH score. The practicality of the SICH score lies in its ease of calculation because it requires only review of the NCCT to determine its categorization (high probability, indeterminate, low probability) and clinical data routinely obtained on presentation to the emergency department (patient age, sex, history of hypertension, use of antiplatelet medications, platelet count, INR, and aPTT).

The SICH score can be rapidly calculated immediately after the NCCT examination has been performed while the patient is still on the CT scanner table, and could serve as a valuable tool in the clinical decision of whether to perform neurovascular evaluation. Therefore, the SICH score may be most useful at institutions in which neurovascular evaluation is not routinely performed on all patients with $\mathrm{ICH}$ but is reserved for those patients who are deemed most likely to harbor an underlying vascular abnormality. For example, at these institutions, patients with a SICH score of zero would not merit neurovascular evaluation, patients with $\mathrm{SICH}$ scores of 1-2 

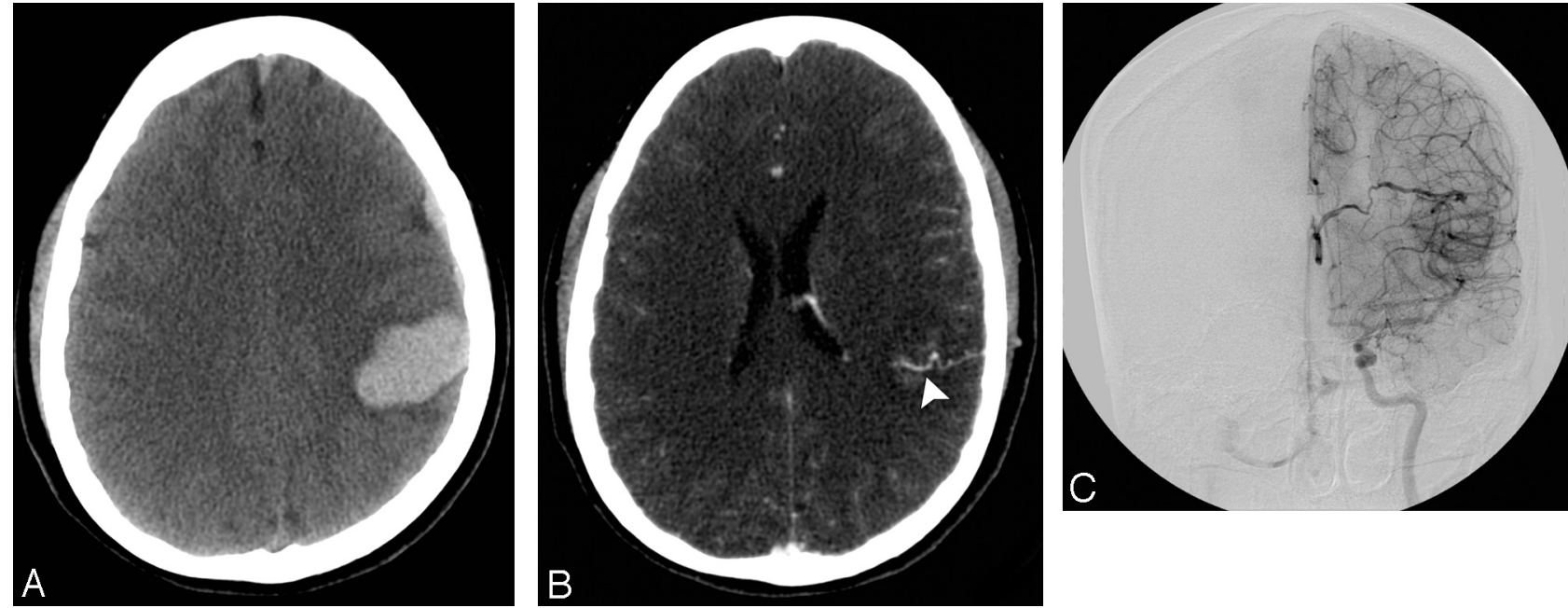

Fig 4. A 44-year-old woman without history of hypertension and with intact coagulation presented with headache. A, Indeterminate NCCT scan demonstrates a left parietal ICH (SICH score, 5). There was associated subdural hemorrhage overlying the left frontal lobe but no associated IVH or SAH. B, CTA source image demonstrates an abnormal vessel along the inferior aspect of the ICH in the left parietal lobe, consistent with an AVF (arrowhead). C. Frontal left internal carotid artery catheter angiogram confirms the presence of a left parietal AVF with deep venous drainage into the left internal cerebral vein.
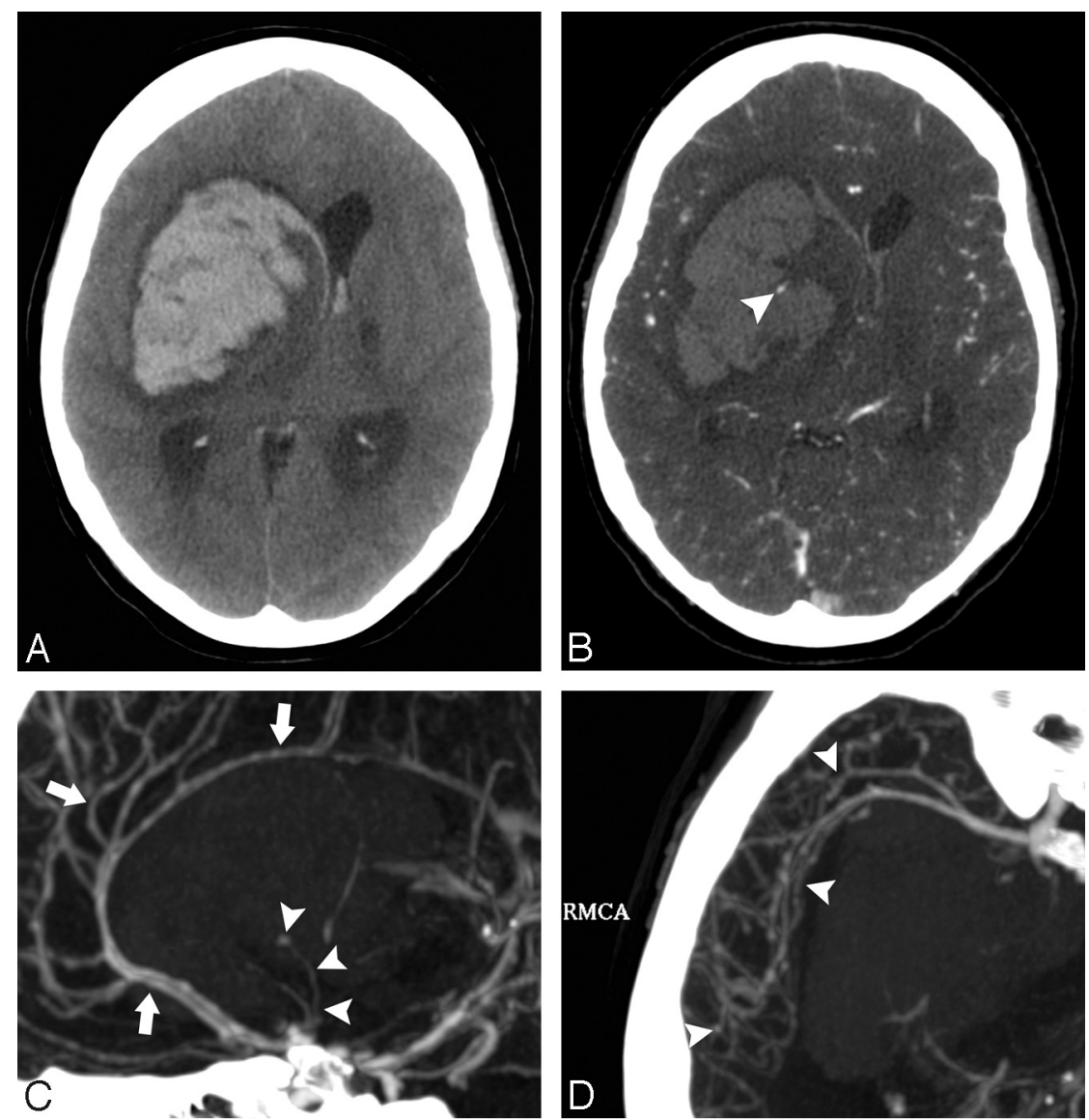

Fig 5. A 50-year-old woman without a history of hypertension and with intact coagulation presented with acute onset of unresponsiveness. A, Low-probability NCCT scan demonstrates an acute right basal ganglia ICH with associated IVH (SICH score, 3). B, CTA source image demonstrates a 3-mm outpouching arising from a lenticulostriate branch of the right middle cerebral artery (arrowhead), consistent with an aneurysm. C, CTA MIP image in the sagittal plane redemonstrates the right lenticulostriate aneurysm (arrowheads) as well as a diffuse luminal irregularity in the visualized anterior cerebral artery branches (arrows). D, CTA MIP image in the axial plane demonstrates diffuse luminal irregularity in the right middle cerebral artery branches (arrowheads). These findings are consistent with vasculitis with secondary pseudoaneurysm formation and rupture. The patient was ultimately found to have Lyme disease affecting the central nervous system.

could be initially screened with a noninvasive technique such as MDCTA and would only undergo further evaluation with conventional catheter angiography if the initial CTA is either positive or equivocal, and patients with SICH scores $\geq 3$ could be evaluated directly with conventional catheter angiography.

However, some institutions (including our own) consider 

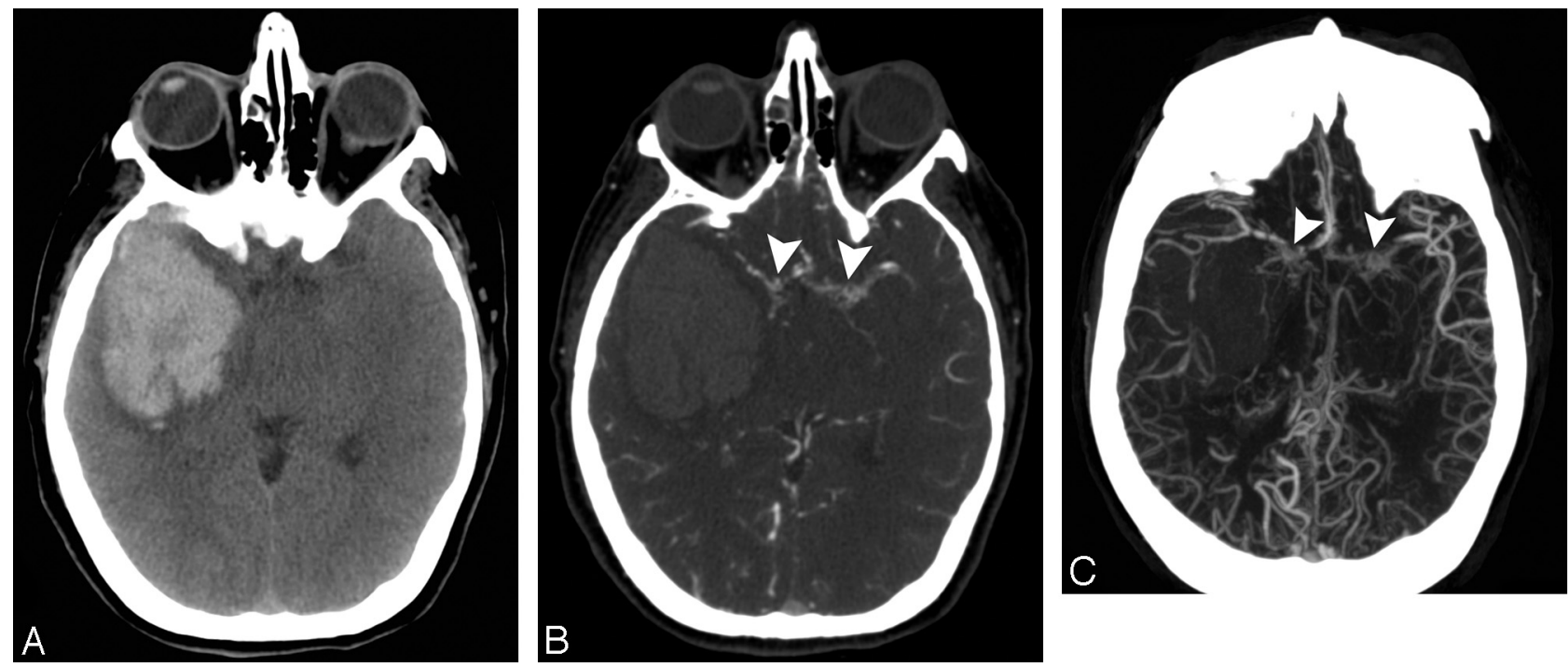

Fig 6. A 42-year-old woman without a history of hypertension and with intact coagulation presented with severe headache. A, Indeterminate NCCT scan demonstrates an acute right temporal ICH without associated IVH or SAH (SICH score, 5). B, CTA source image demonstrates occlusion of the supraclinoid segments of the internal carotid arteries and proximal M1 segments of the middle cerebral arteries bilaterally, with numerous associated lenticulostriate collateral vessels (arrowheads), consistent with Moyamoya phenomenon. C, CTA MIP image in the axial plane redemonstrates the findings of Moyamoya phenomenon (arrowheads).

\begin{tabular}{lc}
\hline Table 2: Calculation of the SICH score & \\
\hline Parameter & Points \\
\hline NCCT categorization & \\
High probability & 2 \\
Indeterminate & 1 \\
Low probability & 0 \\
Age group & 2 \\
18-45 years & 1 \\
$46-70$ years & 0 \\
$\geq 71$ years & \\
Sex & 1 \\
Female & 0 \\
Male & \\
Neither known HTN nor impaired coagulation & \\
Yes & 1 \\
No & 0
\end{tabular}

Note:-The SICH score is calculated by adding the total number of points for a given patient.

a High-probability NCCT: an examination with either 1) enlarged vessels or calcifications along the margins of the $\mathrm{ICH}$ or 2) hyperattenuation within a dural venous sinus or cortical vein along the presumed venous drainage path of the ICH. Low-probability NCCT: an examination in which neither 1) nor 2) is present and the ICH is located in the basal ganglia, examination in which neither 1) nor 2) is present and the ICH is located in the basal ganglia,
thalamus, or brain stem. Indeterminate NCCT: an examination that does not meet criteria thalamus, or brain stem. Indeterminate
for a high- or low-probability NCCT.

Impaired coagulation defined as admission INR $>3$, aPTT $>80$ seconds, platelet count $<50,000$, or daily antiplatelet therapy.

a positivity rate of $>10 \%$ for a low-risk noninvasive diagnostic test such as MDCTA to be high enough to merit performing it in all patients. Furthermore, at our institution (and others around the world), the presence of a "spot sign" is increasingly being used as a valuable tool for the prediction of hematoma expansion and poor clinical outcome in patients with primary ICH. ${ }^{24-32}$ Hence, the usefulness of the SICH score at institutions like our own may lie in selecting patients with ICH for more invasive diagnostic tests such as conventional catheter angiography when the initial MDCTA examination is either negative or equivocal. For example, at these institutions, patients with low SICH scores (0-2) would only merit further evaluation with conventional catheter angiography if the initial CTA is either positive or equivocal, while patients with high SICH scores $(\geq 3)$ would merit further evaluation with conventional catheter angiography even if the initial CTA is negative.

The diagnostic approaches proposed above would minimize the overall number of negative conventional catheter angiograms performed in this patient population while also minimizing the number of patients with underlying vascular abnormalities who are not diagnosed correctly. This, in turn, would result in decreased radiation exposure in this patient population as well as reduced exposure to iodinated contrast material in patients with $\mathrm{ICH}$ and renal insufficiency or failure.

The limitations of the our study are the retrospective nature of the derivation cohort of the SICH score, the potential selection bias generated by the inclusion of only patients who presented with ICH and were evaluated with MDCTA, and the lack of independent validation of this scoring system. Although currently at our institution, most patients with nontraumatic ICH are evaluated with MDCTA on admission to assess for the presence of an underlying vascular etiology as well as a "spot sign," in the retrospective cohort only approximately half of patients with nontraumatic ICH were evaluated with MDCTA on admission because patients who, on clinical grounds, were deemed unlikely to harbor an underlying vascular etiology for the ICH (likely those with low SICH scores) did not undergo MDCTA evaluation. However, the similar CTA positivity rates in each $\mathrm{SICH}$ score group and the similar AUCs for the scoring system in both patient cohorts provide evidence that this selection bias may not be significant. Nevertheless, the incidence of underlying vascular etiologies in our patient population, particularly in patients with low SICH scores, may be lower than reported in this study.

\section{Conclusions}

The SICH score successfully predicts a given ICH patient's risk of harboring an underlying vascular etiology. This practical scoring system could be used as a guide for selecting patients 


\begin{tabular}{|c|c|c|c|c|c|c|}
\hline \multirow[b]{2}{*}{ Score } & \multicolumn{2}{|c|}{$\begin{array}{l}\text { Retrospective-Derivation Cohort } \\
\qquad(n=623)\end{array}$} & \multicolumn{2}{|c|}{$\begin{array}{l}\text { Prospective-Validation Cohort } \\
\qquad(n=222)\end{array}$} & \multicolumn{2}{|c|}{ All Patients $(n=845)$} \\
\hline & No. $(\%)$ & $\begin{array}{l}\% \text { Positive } \\
\text { CTAs }\end{array}$ & No. $(\%)$ & $\begin{array}{l}\% \text { Positive } \\
\text { CTAs }\end{array}$ & No. $(\%)$ & $\begin{array}{l}\% \text { Positive } \\
\text { CTAs }\end{array}$ \\
\hline 0 & $37(5.9)$ & 0 & $15(6.8)$ & 0 & $52(6.1)$ & 0 \\
\hline 1 & $145(23.3)$ & 1.4 & $67(30.2)$ & 1.5 & $212(25.1)$ & 1.4 \\
\hline 2 & $209(33.5)$ & 5.3 & $68(30.6)$ & 4.4 & 277 (32.8) & 5.1 \\
\hline 3 & $138(22.2)$ & 18.1 & $40(18.0)$ & 20 & $178(21.1)$ & 18.5 \\
\hline 4 & $61(9.8)$ & 39.3 & $21(9.5)$ & 38.1 & $82(9.7)$ & 39 \\
\hline 5 & $28(4.5)$ & 85.7 & $10(4.5)$ & 80 & $38(4.5)$ & 84.2 \\
\hline 6 & $5(0.8)$ & 100 & $1(0.4)$ & 100 & $6(0.7)$ & 100 \\
\hline AUC (95\% CI) & & $0.86(0.83-0.89)$ & & $0.87(0.82-0.91)$ & & $0.87(0.84-0.89)$ \\
\hline MOP & & $>2$ & & $>2$ & & $>2$ \\
\hline Sensitivity & & 85.7 & & 86.2 & & 85.8 \\
\hline Specificity & & 71.1 & & 75.6 & & 72.3 \\
\hline$P$ value & & $<.0001$ & & $<.0001$ & & $<.0001$ \\
\hline
\end{tabular}

with ICH for neurovascular evaluation to exclude the presence of a vascular abnormality as the $\mathrm{ICH}$ etiology. Independent validation of this scoring system is necessary.

\section{Acknowledgments}

We thank Eleni K. Balasalle for her contribution to the artwork for this manuscript.

\section{References}

1. Qureshi AI, Tuhrim S, Broderick JP, et al. Spontaneous intracerebral hemorrhage. N Engl J Med 2001;344:1450-60

2. Badjatia N, Rosand J. Intracerebral hemorrhage. Neurologist 2005;11:311-24

3. Singh $\mathrm{T}$, Chakera T. Dural sinus thrombosis presenting as unilateral lobar haematomas with mass effect: an easily misdiagnosed cause of cerebral haemorrhage. Australas Radiol 2002;46:351-65

4. Jane JA, Kassell NF, Torner JC, et al. The natural history of aneurysms and arteriovenous malformations. J Neurosurg 1985;62:321-23

5. The Arteriovenous Malformation Study Group. Arteriovenous malformations of the brain in adults. N Engl J Med 1999;340:1812-18

6. Ondra SL, Troupp H, George ED, et al. The natural history of symptomatic arteriovenous malformations of the brain: a 24-year follow-up assessment. J Neurosurg 1990;73:387-91

7. Yeung R, Ahmad T, Aviv RI, et al. Comparison of CTA to DSA in determining the etiology of spontaneous ICH. Can J Neurol Sci 2009;36:176-80

8. Romero JM, Artunduaga M, Forero NP, et al. Accuracy of CT angiography for the diagnosis of vascular abnormalities causing intraparenchymal hemorrhage in young patients. Emerg Radiol 2009;16:195-201

9. Yoon DY, Chang SK, Choi CS, et al. Multidetector row CT angiography in spontaneous lobar intracerebral hemorrhage: a prospective comparison with conventional angiography. AJNR Am J Neuroradiol 2009;30:962-67

10. Delgado Almandoz JE, Schaefer PW, Forero NP, et al. Diagnostic accuracy and yield of multidetector CT angiography in the evaluation of spontaneous intraparenchymal cerebral hemorrhage. AJNR Am J Neuroradiol 2009;30:1213-21

11. Toffol GJ, Biller J, Adams HP, et al. The predicted value of arteriography in nontraumatic intracerebral hemorrhage. Stroke 1986;17:881-83

12. Loes DJ, Smoker WR, Biller J, et al. Nontraumatic lobar intracerebral hemorrhage: CT/angiographic correlation. AJNR Am J Neuroradiol 1987;8:1027-30

13. Laissy JP, Normand G, Monroe M, et al. Spontaneous intracerebral hematomas from vascular causes: predictive value of CT compared with angiography. Neuroradiology 1991;33:291-95

14. Halpin SF, Britton JA, Byrne JV, et al. Prospective evaluation of cerebral angiography and computed tomography in cerebral haematoma. J Neurol Neurosurg Psych 1994;57:1180-86

15. Zhu XL, Chan MS, Poon WS. Spontaneous intracranial hemorrhage: which patients need diagnostic cerebral angiography? A prospective study of 206 cases and review of the literature. Stroke 1997;28:1406-09
16. Griffiths PD, Beveridge CJ, Gholkar A. Angiography in non-traumatic brain haematoma: an analysis of 100 cases. Acta Radiol 1997;38:797-802

17. Ruiz-Sandoval JL, Cantu C, Barinagarrementeria F. Intracerebral hemorrhage in young people: analysis of risk factors, location, causes and prognosis. Stroke 1999;30:537-41

18. Ohtani R, Kazui S, Tomimoto H, et al. Clinical and radiographic features of lobar cerebral hemorrhage: hypertensive versus non-hypertensive cases. Intern Med 2003;42:576-80

19. Matsumoto K, Sakaki S, Abekura M, et al. Co-existence of unruptured cerebral aneurysms in patients with hypertensive intracerebral hemorrhage. Acta Neurochir (Wien) 2004;146:1085-89

20. Abu Bakar I, Shuaib IL, Mohd Ariff AR, et al. Diagnostic cerebral angiography in spontaneous intracranial haemorrhage: a guide for developing countries. Asian J Surg 2005;28:1-6

21. Cordonnier C, Klijn CJ, van Beijnum J, et al. Radiological investigation of spontaneous intracerebral hemorrhage. systematic review and trinational survey. Stroke 2010;41:685-90. Epub 2010 Feb 18

22. Knudsen KA, Rosand J, Karluk D, et al. Clinical diagnosis of cerebral amyloid angiopathy: validation of the Boston criteria. Neurology 2001;56:537-39

23. Fang MC, Chang Y, Hylek EM, et al. Advanced age, anticoagulation intensity, and risk for intracranial hemorrhage among patients taking warfarin for atrial fibrillation. Ann Intern Med 2004;141:745-52

24. Becker KJ, Baxter AB, Bybee HM, et al. Extravasation of radiographic contrast is an independent predictor of death in primary intracerebral hemorrhage. Stroke 1999;30:2025-32

25. Wada R, Aviv RI, Fox AJ, et al. CT angiography “spot sign" predicts hematoma expansion in acute intracerebral hemorrhage. Stroke 2007;38:1257-62

26. Goldstein JN, Fazen LE, Snider R, et al. Contrast extravasation on CT angiography predicts hematoma expansion in intracerebral hemorrhage. Neurology 2007;68:889-94

27. Kim J, Smith A, Hemphill JC III, et al. Contrast extravasation on CT predicts mortality in primary intracerebral hemorrhage. AJNR Am J Neuroradiol 2008;29:520-25

28. Ederies A, Demchuk A, Chia T, et al. Postcontrast CT extravasation is associated with hematoma expansion in CTA spot negative patients. Stroke 2009;40:1672-76

29. Thompson AL, Kosior JC, Gladstone DJ, et al, for the PREDICTS/Sunnybrook ICH CTA Study Group. Defining the CT angiography 'spot sign' in primary intracerebral hemorrhage. Can J Neurol Sci 2009;36:456-61

30. Delgado Almandoz JE, Yoo AJ, Stone MJ, et al. Systematic characterization of the computed tomography angiography spot sign in primary intracerebral hemorrhage identifies patients at highest risk for hematoma expansion: the spot sign score. Stroke 2009;40:2994-3000

31. Delgado Almandoz JE, Yoo AJ, Stone MJ, et al. The spot sign score in primary intracerebral hemorrhage identifies patients at highest risk of in-hospital mortality and poor outcome among survivors. Stroke 2010;41:54-60

32. Hallevi H, Abraham AT, Barreto AD, et al. The spot sign in intracerebral hemorrhage: the importance of looking for contrast extravasation. Cerebrovasc Dis 2010;29:217-20 